\title{
Ultrasound-guided Microwave Ablation of Adenolymphoma of Parotid Gland: A Case Report
}

\author{
Xiaohui Ji, MD ${ }^{a, ~}$, Juanjuan Li, MD ${ }^{a}$, Dongxia Gao, MD \\ ${ }^{a}$ Department of Ultrasound, The fourth Hospital of Hebei Medical University, Hebei, China \\ Received May12, 2020; revision received June 4, 2020; accepted June7, 2020
}

\begin{abstract}
Adenolymphoma is the second most common benign parotid tumor after pleomorphic adenoma. Although microwave ablation (MWA) has been proved to be effective for benign breast and thyroid nodules, there are few studies on ablation of adenolymphoma of parotid gland. An 82-year-old male with adenolymphoma of bilateral parotid gland treated with ultrasoundguided MWA. After 12 months of follow-up, the volume of bilateral parotid nodules was significantly reduced without recurrence and obvious complications. In conclusion, ultrasound-guided MWA is a safe and effective method for patients with parotid adenolymphoma who cannot tolerate surgery.
\end{abstract}

Key words: Adenolymphoma;Parotid gland; Microwave ablation; Ultrasound-guided; Contrast-enhanced US

Advanced Ultrasound in Diagnosis and Therapy 2022; 01: 022-025

DOI: 10.37015/AUDT.2021.200046

$\mathrm{A}$ denolymphoma, also known as Warthin tumor, is the second most common benign tumor of the parotid next only to pleomorphic adenoma[1]. Of all benign parotid tumors, 25\% are diagnosed as adenolymphoma [2]. Warthin tumor is slow growing and there appears to be related to severe smoking and bilateral Warthin tumors.Ipsilateral multi-centric occurrence is a well-documented feature of the disease [3]. Warthin tumors have a tendency to occur in the parotid tail where the majority of parotid lymph nodes occur, so partial parotidectomy is often required although ideal extent of surgical intervention for adenolymphoma of the parotid gland remains a matter of controversy $[1,4]$. In recent years, with MWA widely applied for thyroid and breast nodules whether MWA can be used as an alternative treatment for Warthin tumor patients has aroused our interest. Here, we report a case of Warthin tumor of bilateral parotid gland, which was successfully treated by ultrasound-guided MWA.

\section{Case Report}

An 82-year-old male patient was admitted to our hospital due to the discovery of right parotid gland mass for more than 40 days. 40 days ago, the patient inadvertently found a "small jujube" size tumor behind the right ear without any treatment, and then the tumor gradually increased to the size of "walnut" with occasional discomfort. Physical examination showed the right parotid firm mass $(3.5 \mathrm{~cm} \times 3.0 \mathrm{~cm} \times 2.0 \mathrm{~cm})$ and the left parotid firm mass $(1.5 \mathrm{~cm} \times 1.0 \mathrm{~cm} \times 1.0 \mathrm{~cm})$ were medium hard, clear boundaries, mobility, with no overlying skin changes and non-tender. The facial nerve function was normal, and there was no other palpable mass in neck examination.

Preoperative computed tomography (CT) scan of the head and neck showed a $1.9 \mathrm{~cm} \times 1.4 \mathrm{~cm}$ fairly well marginated inhomogeneous enhancing lesion within the right parotid gland and $1.0 \mathrm{~cm} \times 0.8 \mathrm{~cm}$ fairly well marginated distinctly enhancing lesion within the left. Preoperative ultrasound (US) of the parotid showed solid-cystic nodules in the bilateral (right and left) parotid, $2.7 \mathrm{~cm} \times 1.6 \mathrm{~cm} \times 1.5 \mathrm{~cm}\left(3.39 \mathrm{~cm}^{3}\right)$ and $2.3 \mathrm{~cm} \times 1.2 \mathrm{~cm} \times 1.0 \mathrm{~cm}\left(1.44 \mathrm{~cm}^{3}\right)$ respectively, regular shaped and fairly well marginated (Fig.1). US-guided core needle biopsy was performed subsequently and

\footnotetext{
* Corresponding author:Department of Ultrasound, The fourth Hospital of Hebei Medical University, Tianshan Street 169 , Yuhua District, Shijiazhuang, Hebei, China.

e-mail: hbjixiaohui@163.com

2576-2508/O AUDT 2022 • http://www.AUDT.org

This is an open-access article distributed under the terms of the Creative Commons Attribution 4.0 International license, which permits unrestricted use, distribution and reproduction in any medium provided that the original work is properly attributed.
} 
adenolymphoma was confirmed by pathology.

In view of the older patients and more underlying diseases, US-guided MWA was considered. The patient signed the informed consent.
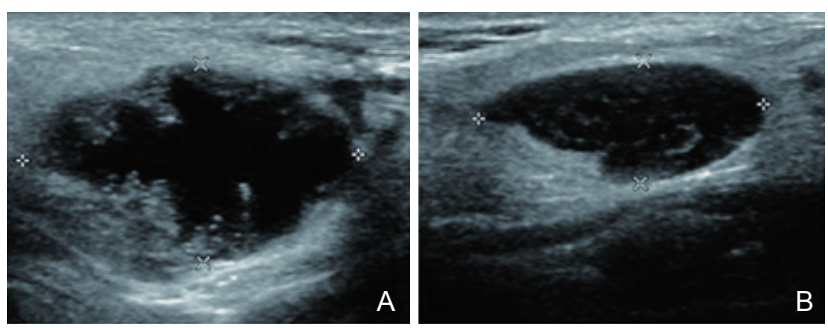

Figure 1 US evaluation before MWA. (A, B) Longitudinal ultrasound images showed that there was a mix nodule of $2.7 \mathrm{~cm} \times 1.6 \mathrm{~cm} \times 1.5 \mathrm{~cm}$ in the right parotid gland and $2.3 \mathrm{~cm} \times 1.2 \mathrm{~cm} \times 1.0 \mathrm{~cm}$ in the left side, with regular shape and clear boundary.

US and contrast-enhanced US (Phillips IU22, Holland), with a $5-12 \mathrm{MHz}$ linear array transducer, were applied to evaluate the lesions of bilateral parotid before MWA by a sonographer with 3 years of experience in MWA for breast and thyroid nodules. The contrast agent (2.4ml, SonoVue, Bracco, Switzerland) entered in the parotid nodules of both sides at 20s and the nodular enhancement was visible in solid area (Fig. 2). With patient placed in lateral position, local anesthesia with $1 \%$ lidocaine was performed on the puncture point. Then the puncture pointof skin pricked by 16-guage needle, under ultrasound-guided, a mixture of $2 \%$ lidocaine and $0.9 \%$ normal saline was infused into the surrounding parotid capsule, as the so-called "hydrodissection technique" to provide a safe barrier avoiding thermal damage to adjacent structures. With microwave ablation instrument (Nanjing Yigao Microwave Electric Institute, Nanjing, China), a 16-gauge MWA antenna was inserted into the nodule along its the longest diameter and positioned in the designated place. The microwave system was $2450 \mathrm{MHz}$, and output power $30 \mathrm{~W}$ was selected in the study. The ablation procedures were monitored by realtime US, and ablations were not terminated until the transient hyperechoic zone caused by the gas covered all units of the nodules. The efficacy of MWA was evaluated by contrast-enhanced US immediately after MWA. No contrast enhancement was found in bilateral parotid nodules. The patient was well tolerated during the operation, and was given local ice compress at the puncture point after the operation without taking any drugs. The general condition was observed continuously for 24 hours. Slight auricle numbness and no facial nerve injury were found after operation.
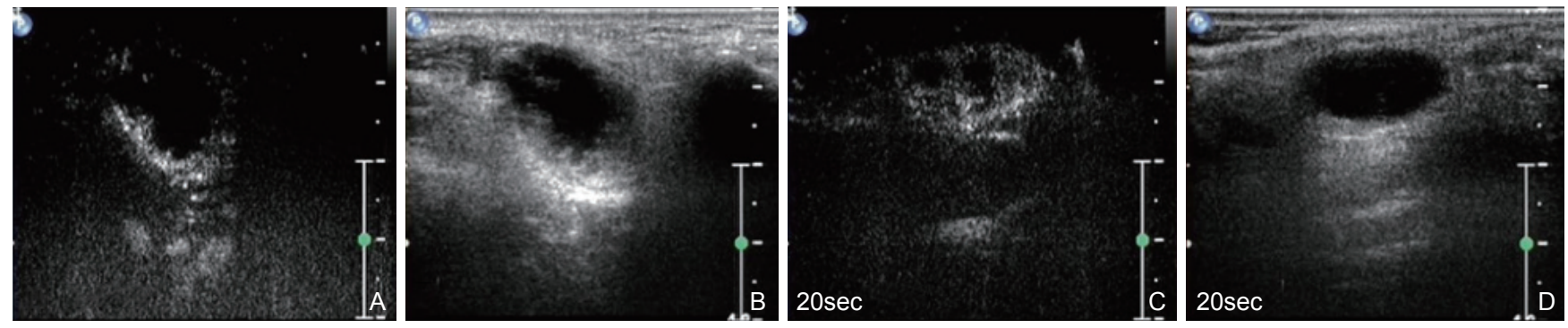

Figure 2 Contrast-enhanced US of the nodule in the bilateral parotid before MWA. (A, B) The solid area of the inner wall of the right nodule was rapidly and highly enhanced; (C, D) Most of the left nodule were enhanced and some areas in the center were not.

The outcomes were assessed by US and contrastenhanced US examination. After one month of MWA, the right parotid nodule was $1.9 \mathrm{~cm} \times 1.4 \mathrm{~cm} \times 1.2 \mathrm{~cm}\left(1.67 \mathrm{~cm}^{3}\right)$ and the left one was $1.7 \mathrm{~cm} \times 1.2 \mathrm{~cm} \times 1.1 \mathrm{~cm}\left(1.17 \mathrm{~cm}^{3}\right)$, with regular shape and clear boundaries (Fig. 3 A, B), but no enhancement (Fig. 3 E-H). The nodules volume decreased from $3.39 \mathrm{~cm}^{3}$ to $1.67 \mathrm{~cm}^{3}$ (right) and $1.44 \mathrm{~cm}^{3}$ to $1.17 \mathrm{~cm}^{3}$ (left) at the 1-month follow-up, which the volume reduction rate (VRR) was $50.7 \%$ and $18.8 \%$, respectively (Fig. 4A, B). After 6 months of MWA, the ablation areas shrunk to $1.3 \mathrm{~cm} \times 0.8 \mathrm{~cm} \times 0.7 \mathrm{~cm}\left(0.38 \mathrm{~cm}^{3}\right.$, right $)$ and $1.3 \mathrm{~cm} \times 0.6 \mathrm{~cm} \times 0.5 \mathrm{~cm}\left(0.20 \mathrm{~cm}^{3}\right.$, left $)$, respectively, with a well-defined margin (Fig. 3 C, D). The nodules volume decreased significantly from $3.39 \mathrm{~cm}^{3}$ to $0.38 \mathrm{~cm}^{3}$ (right) and $1.44 \mathrm{~cm}^{3}$ to $0.20 \mathrm{~cm}^{3}$ (left) at the 6 -month follow-up, with the VRR of $88.8 \%$ and $86.1 \%$, respectively (Fig. 4 A, B). No facial nerve injury and other complications were found in this case. No recurrence was found in the follow-up of 6 months. After 9 months of MWA treatment, CT scan of the neck showed that $0.8 \mathrm{~cm} \times 0.7 \mathrm{~cm}$ nodule was found in the right parotid gland and $0.6 \mathrm{~cm} \times$ $0.5 \mathrm{~cm}$ nodule was seen in the left side, regular shape, clear boundary, but no enhancement. After 12 months of MWA, the lesions of bilateral parotid detected by US and $\mathrm{CT}$ were not obvious because of absorption. The patient is still in continuous follow-up.

\section{Discussion}

Surgery is a mature and effective method for the treatment of Warthin tumor, although the ideal range of 

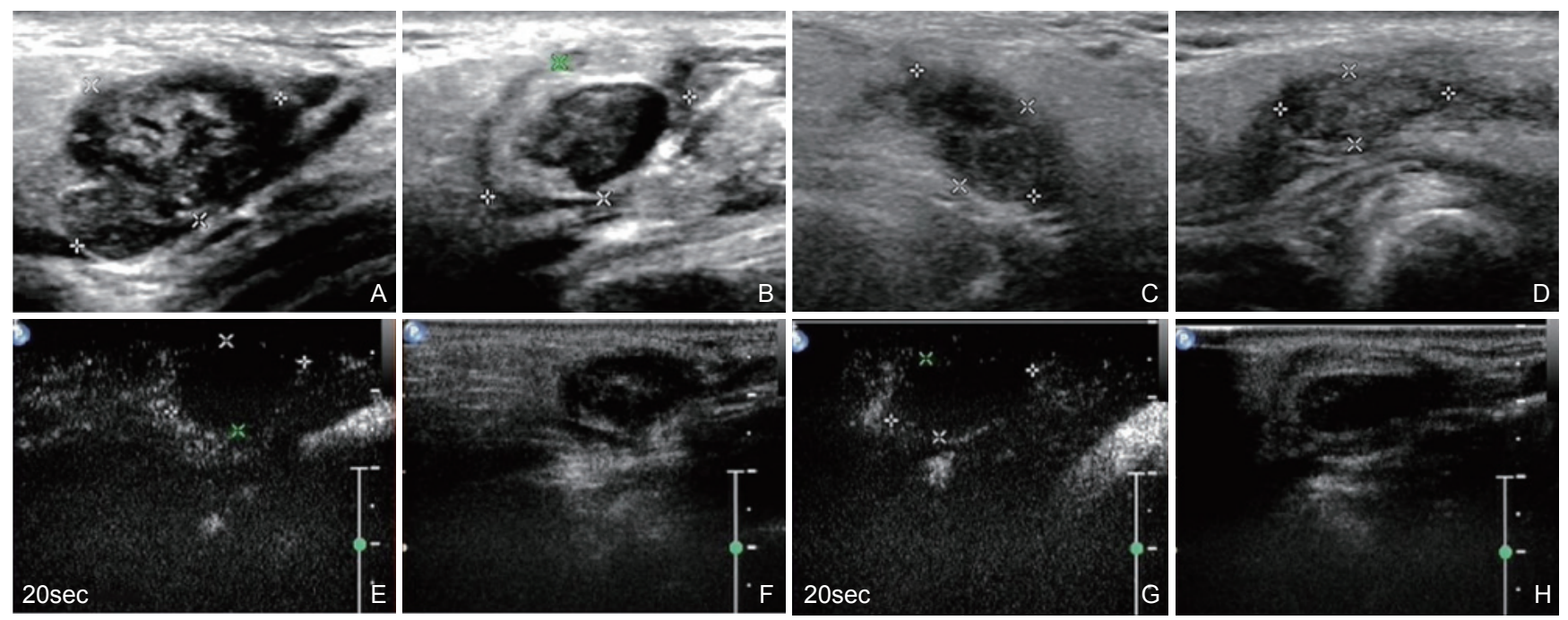

Figure 3 Follow-up of the patient after MWA. (A, B) One month after MWA, the volume of the right parotid nodule shrank to $1.9 \mathrm{~cm} \times 1.4 \mathrm{~cm} \times 1.2 \mathrm{~cm}$ and the left shrank to $1.7 \mathrm{~cm} \times 1.2 \mathrm{~cm} \times 1.1 \mathrm{~cm}$ with a well-defined margin; $(C, D)$ Six months after MWA, the volume of the right parotid nodule shrank to $1.3 \mathrm{~cm} \times 0.8 \mathrm{~cm} \times 0.7 \mathrm{~cm}$ and the left shrank to $1.3 \mathrm{~cm} \times 0.6 \mathrm{~cm} \times 0.5 \mathrm{~cm} ;(\mathrm{E}, \mathrm{F}, \mathrm{G}, \mathrm{H})$ One month after MWA, contrast-enhanced US showed no enhancement in the bilateral nodules.

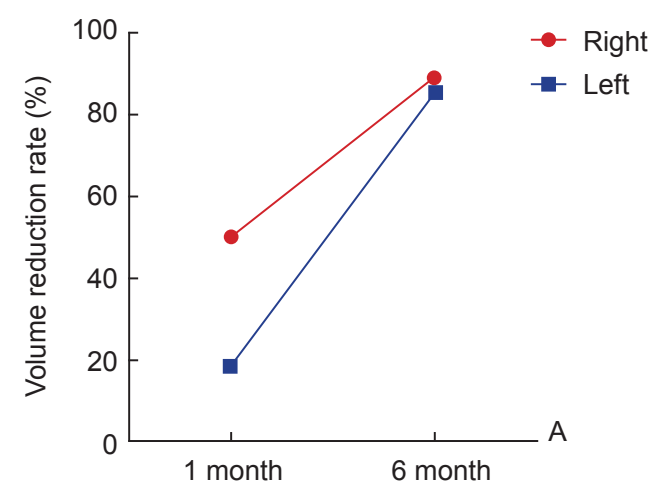

Figure 4 The curve of nodule volume with time after MWA.

surgical treatment for Warthin tumor is still controversial, mainly because it has the risk of bleeding, postoperative infection, permanent facial nerve injury and recurrence [4,5]. Although MWA is effective for benign breast nodules and thyroid nodules, with good VRR and treatment success rate $(77.2 \%$ and $97 \%$ at 6 months, $81.6 \%$ and $100 \%$ at 12 months, respectively) [6], it is rarely used in parotid nodules. Considering that this case is an elderly patient with many underlying diseases and high risk of surgery and anesthesia, MWA was selected as the treatment method after full informed consent to reduce invasion and improve safety. The advantage of MWA is that it can be performed in a short time under local anesthesia, reach a higher intratumor temperature in the ablation area instantaneously, and use multiple therapeutic probes for larger lesions [7]. The risk of facial nerve injury should also be considered when the ablation process is guided by real-time US. In this case, the bilateral parotid nodules were located under the ear,

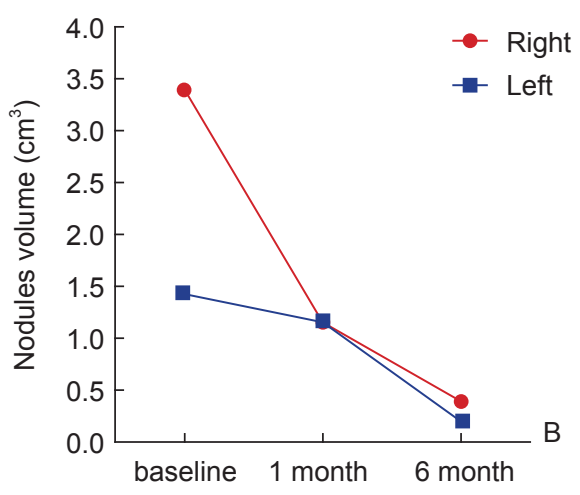

avoiding the normal facial nerve area, so there was no facial nerve injury after MWA.

Maurilio D et al. found that there was a significant correlation between different ultrasound features in radiofrequency ablation of thyroid nodules (sponge nodule showed $76 \%$ vs $67 \%$ and $66 \%$ reduction in mixed nodule and solid nodule, respectively, $P<0.01$ ) and vascular (abundant peripheral and internal blood supply were $71 \%$ vs $68 \%$ and $67 \%$ reduction of slight peripheral/internal blood supply and peripheral blood supply, respectively, $P<0.03$ ), but there was no large calcification [8]. In addition, there was a slight negative correlation between nodule base volume and contraction rate. Preoperative ultrasound showed that bilateral parotid nodules were cystic and solid nodules with large volume. One month after the operation, the volume of the right and left nodules decreased by $50.7 \%$ and $18.8 \%$, respectively. Six months after the operation, the volume of the right and left nodules decreased by $88.8 \%$ 
and $86.1 \%$, respectively. As reported in the research, the VRR here is similar to that of thyroid nodules after MWA. In this case, the VRR of the right side is larger than that of the left side, mainly because the cystic ratio of the right side is larger than that of the left side. After 12 months follow-up, US and enhanced CT showed no enhancement and recurrence of the nodules, indicating that MWA is safe and effective in the treatment of parotid nodules.

\section{Conclusion}

In summary, US-guided MWA, a minimally invasive alternative to surgery, is a promising tool for the management of nodules of parotid gland with low incidence of local complications and good therapeutic effect. More cases and multi-center studies are needed to verify the effectiveness of US-guided MWA in the treatment of parotid adenolymphoma or parotid nodules, and to determine whether we can safely take the next step along with the path of minimally invasive treatment.

\section{Conflict of Interest}

The authors declare that they have no competing interests.

\section{References}

[1] Pötzl T, Iselin S, Husner A. Raumforderung der glandulaparotis [Tumor of the parotid gland]. Praxis 2016;105:577-579. [In German].

[2] Patel DK, Morton RP. Demographics of benign parotid tumours: Warthin' stumour versus other benign salivary tumours. Acta Otolaryngol 2016; 136: 83-86.

[3] Sagiv D, Witt RL, Glikson E, Mansour J, Shalmon B, Yakirevitch A, et al. Warthin tumor within the superficial lobe of the parotid gland: a suggested criterion for diagnosis. Eur Arch Otorhinolaryngol 2017; 274: 1993-1996.

[4] Mantsopoulos K, Goncalves M, Koch M, Iro H. Extracapsular dissection for Warthin tumors despite the risk of ipsilateral metachronous occurrence. Laryngoscope 2018; 128: 2521-2524.

[5] Kadletz L, Grasl S, Grasl MC, Perisanidis C, Erovic BM. Extracapsular dissection versus superficial parotidectomy in benign parotid gland tumors: the Vienna medical school experience. Head Neck 2017; 39: 356-360.

[6] Yue WW, Wang SR, Lu F, Sun LP, Guo LH, Zhang YL, et al. Radiofrequency ablation vs. microwave ablation for patients with benign thyroid nodules: a propensity score matching study. Endocrine 2017; 55: 485-495.

[7] Fleming MM, Holbrook AI, Newell MS. Update on image-guided percutaneous ablation of breast cancer. AJR Am J Roentgenol 2017; 208: 267-274.

[8] Deandrea M, Garino F, Alberto M, Garberoglio R, Rossetto R, Bonelli N, et al. Radiofrequency ablation for benign thyroid nodules according to different ultrasound features: an Italian multicentre prospective study. Eur J Endocrinol 2019; 180: 79-87. 